Piotr Adamski ${ }^{1}$, Małgorzata Ostrowska ${ }^{1}$, Wiktor Dariusz Sroka ${ }^{2}$, Michał Piotr Marszałt ${ }^{2}$ Emilia Kolasińska ${ }^{3}$, Paulina Lisiecka ${ }^{3}$, Natalia Skibińska ${ }^{3}$, Przemysław Sobczak ${ }^{3}$, Paulina Szarwas ${ }^{3}$, Jacek Kubica ${ }^{4}$, Marek Koziński ${ }^{1}$

\title{
Does morphine administration affect ticagrelor conversion to its active metabolite in patients with acute myocardial infarction? A sub-analysis of the randomized, double-blind, placebo- -controlled IMPRESSION trial
}

\section{Corresponding author:}

Piotr Adamski, MD

Department of Principles of Clinical Medicine,

Collegium Medicum, Nicolaus

Copernicus University

9 Skłodowskiej-Curie Street,

85-094 Bydgoszcz, Poland

Tel.: + 48525854023

Fax: +48525854024

E-mail: piotr.adamski@wp.eu
Folia Medica Copernicana 2015; Volume 3, Number 3, 100-106 10.5603/FMC.2015.0003 Copyright (C) 2015 Via Medica ISSN 2300-5432

\begin{abstract}
Background. Therapy with aspirin and one of the platelet P2Y12 receptor inhibitors, preferably ticagrelor or prasugrel, is the mainstay of acute myocardial infarction (AMI) treatment. Morphine is the most commonly used analgesic in AMI patients. The IMPRESSION study was the first randomized trial to confirm that morphine use in this clinical setting leads to a delayed and attenuated exposure to ticagrelor and its active metabolite (AR-C124910XX). The mechanism underlying this drug-drug interaction remains hypothetical. Material and methods. A post hoc sub-analysis of the IMPRESSION study, a phase IV, single center, randomized, double-blind, placebo-controlled trial, was performed to examine whether morphine administration interferes with the proportion of AR-C124910XX produced from ticagrelor in AMI patients. Pharmacokinetic results of all subjects pretreated with placebo $(n=35)$ and morphine $(n=35)$ were analyzed. The ratio of total exposure to AR-C124910XX to total exposure to ticagrelor for $12 \mathrm{~h}$ was used to illustrate the rate of ticagrelor metabolism. Total exposure to investigated compounds was measured as the area under the plasma concentration-time curve (AUC).

Results. The ratios of $A \cup C_{(0-12)}$ for $A R-C 124910 X X$ to $A U C_{(0-12)}$ for ticagrelor were comparable between morphine and placebo pretreated patients (20.9 [13.9-34.6] v. 24.7 [18.1-29.6] \%; $p=0.58)$. Importantly, visual inspection of the relationship between $\mathrm{AUC}_{(0-12)}$ for $\mathrm{AR}-\mathrm{C} 124910 \mathrm{XX}$ and $\mathrm{AUC}_{(0-12)}$ for ticagrelor revealed that regression lines for the morphine and placebo groups were located closely to each other, with a tendency for superimposing. Additionally, we observed similar values of slope coefficients for both study arms in the linear regression equations illustrating the relationship between $\mathrm{AUC}_{(0-12)}$ for $A R-C 124910 X X$ and $\mathrm{AUC}_{(0-12)}$ for ticagrelor $(0.19[ \pm 0.03]$ v. $0.21[ \pm 0.04]$; $p$ for the statistical significance of both slope coefficients $<0.0001$ ).

Conclusions. In the IMPRESSION study, conversion of ticagrelor to AR-C124910XX in AMI patients was not affected by morphine administration.

Key words: ticagrelor, AR-C124910XX, pharmacokinetics, morphine, myocardial infarction
\end{abstract}

Folia Medica Copernicana 2015; 3 (3): 100-106 


\section{Introduction}

Excessive platelet reactivity is considered one of the key factors predisposing to the occurrence of acute myocardial infarction (AMI) and stent thrombosis. Dual antiplatelet therapy with aspirin and one of the platelet P2Y12 receptor inhibitors remains the cornerstone of AMI treatment [1, 2]. The current guidelines of the European Society of Cardiology recommend the use of ticagrelor or prasugrel in AMI patients [3, 4]. Ticagrelor and prasugrel are preferred over clopidogrel in this setting, and, according to the guidelines, the use of the latter should be restricted to the situations when ticagrelor or prasugrel are not available or are contraindicated [3, 4]. In contrast to prasugrel, therapy with ticagrelor is also advocated in AMI patients managed without coronary revascularization [3, 4]. Despite multiple pharmacokinetic and pharmacodynamic differences among the antiplatelet agents, the common feature of clopidogrel, prasugrel and ticagrelor is their oral route of administration. Cangrelor, the first recently approved intravenous P2Y12 receptor inhibitor, is still not commercially available in many European countries, including Poland.

The superiority of ticagrelor over clopidogrel in patients with acute coronary syndromes has been demonstrated in the Platelet Inhibition and Patient Outcomes (PLATO) trial. In this randomized study, therapy with ticagrelor, when compared with clopidogrel treatment, decreased the rate of major cardiovascular events (a composite of death from vascular causes, myocardial infarction, stroke) and all-cause mortality, without a concomitant increase in the occurrence of overall major bleeding complications [5].

Ticagrelor is an oral, reversible, noncompetitive P2Y12 receptor inhibitor, which is characterized by a potent and uniform antiplatelet action [6]. Contrary to clopidogrel and prasugrel, ticagrelor is an active drug which does not require metabolism to inhibit platelet P2Y12 receptor. Therefore, it exerts its antiplatelet effect immediately after absorption. In addition, ticagrelor is extensively metabolized to about ten compounds. Ticagrelor main active metabolite, AR-C124910XX, which is formed by ticagrelor O-de-ethylation through cytochrome P450 CYP3A4 and CYP3A43A5 enzymes, has a similar $\mathrm{P} 2 \mathrm{Y} 12$ receptor potency as the parent drug and seems to exert antiplatelet action comparable to ticagrelor $[7,8]$. The concentration of AR-C124910XX is reported to be approximately one third of the parent drug [7-9]. A predictable and linear pharmacokinetics of ticagrelor and ARC124910XX have been demonstrated in a wide spectrum of patients, including those with stable coronary artery disease and acute coronary syndromes $[10,11]$. Both, circulating ticagrelor and its active metabolite, undergo $\mathrm{N}$-dealkylation and/or glucuronidation, and are finally eliminated via feces and urine in an inactive state [8].
Pain relief is crucial in the treatment of AMI patients not only for the humane reasons. Effective chest pain alleviation reduces heart rate and blood pressure; therefore, it improves the balance between cardiomiocytes' oxygen demand and supply [12]. Although a few new analgesics have been developed in the recent years, morphine remains the most commonly used painkiller in patients with AMI and is continuously endorsed in the subsequent guidelines for the treatment of acute coronary syndromes [3, 4]. Moreover, the extensive experience in the use of morphine in cardiac intensive care units and emergency departments only assures its position in the everyday practice in this setting. Nevertheless, adverse effects of morphine occur frequently. Respiratory depression, hypotension and impaired propulsive motility of gastrointestinal tract, which may lead to emesis, are only a few examples of the potential side effects of morphine use $[12,13]$. In particular, the influence exerted by morphine on the gastrointestinal tract constitutes the danger of a negative impact on absorption of the orally administered drugs, which may lead to an attenuated and delayed action of those compounds. The above mentioned problem largely concerns patients presenting with AMI who require fast and strong platelet blockade, but often simultaneously need effective analgesic treatment.

The results of our recently published Influence of Morphine on Pharmacokinetics and Pharmacodynamics of Ticagrelor in Patients with Acute Myocardial Infarction (IMPRESSION) study confirmed the alleged drug-drug interaction between ticagrelor and morphine [14]. This study was the first randomized trial to document a delayed and attenuated exposure to ticagrelor and AR-C124910XX in AMI patients caused by intravenous administration of morphine prior to the loading dose of ticagrelor [15]. However, the detailed mechanism underlying this drug-drug interaction remains hypothetical. Therefore, we conducted this post hoc sub-analysis of the pharmacokinetic results of the IMPRESSION study in order to test the hypothesis that morphine administration may affect the rate of ticagrelor to AR-C124910XX metabolism in AMI patients.

\section{Materials and methods}

\section{Study and sub-analysis design}

The IMPRESSION study was a phase IV, single center, randomized, double-blind, placebo-controlled trial which was aimed to examine the impact of morphine on plasma concentrations of ticagrelor and AR-C124910XX, as well as to evaluate morphine's influence on the antiplatelet effect of ticagrelor and its active metabolite [14, 15]. The diagnosis of AMI was made according to the third universal definition of myocardial infarction [16]. 
Briefly, the IMPRESSION study included P2Y12 receptor inhibitor-naive patients, aged 18 to 80 , who have not received morphine during the current AMI. Pain free subjects, patients who required analgesia on admission due to high pain intensity, patients with signs and symptoms of acute heart failure or known contraindications to ticagrelor were not considered for enrollment [14, 15]. The primary endpoint of the IMPRESSION study was the area under the plasma concentration-time curve $\left(\mathrm{AUC}_{(0-12)}\right)$ for ticagrelor during the first $12 \mathrm{~h}$ after administration of the ticagrelor loading dose. Secondary endpoints of the trial, among others, included $\mathrm{AUC}_{(0-12)}$ for AR-C124910XX [14, 15]. The detailed study design, complete inclusion and exclusion criteria, entire list of the study endpoints, sample size calculation and the results were previously described and published [14, 15].

We recruited 74 patients for the IMPRESSION trial. However, a total of 70 patients were included in the primary analysis (one patient had STEMI diagnosis not confirmed and three patients, due to chest pain aggravation, required morphine administration outside the study protocol, which resulted in their exclusion from the further participation in the trial) $[14,15]$. Both ST-segment elevation myocardial infarction (STEMI) and non-ST-segment elevation myocardial infarction (NSTEMI) patients admitted to the study site (Cardiology Clinic, Dr. A. Jurasz University Hospital, Bydgoszcz, Poland) were randomly assigned to one of the study arms. Subjects allocated to the intervention arm received intravenous morphine before they were loaded with $180 \mathrm{mg}$ of ticagrelor. Patients in the control arm were injected with placebo $(0.9 \%$ saline) before they were given a $180 \mathrm{mg}$ ticagrelor loading dose [15].

A total of $70 \mathrm{AMI}$ patients (35 morphine pre-treated patients and 35 placebo pre-treated patients) completed the pharmacokinetic and pharmacodynamic assessment [14]. In the IMPRESSION study, the administration of morphine, as compared with placebo, was associated with the lower total exposure to both ticagrelor and its active metabolite AR-C124910XX within the first $12 \mathrm{~h}$ after the administration of a $180 \mathrm{mg}$ ticagrelor loading dose, as measured by $A \cup C_{(0-12)}$. Morphine decreased the exposure to ticagrelor within the first $12 \mathrm{~h}$ of the treatment by $36 \%(p=0.003)$ and to AR-C124910XX by $37 \%$ ( $p=0.008)$. Moreover, the patients in the morphine arm required more time to reach maximal plasma concentration of ticagrelor compared with the subjects from the placebo arm (4 [3-12] v. 2 [2-4] h; p = 0.004) and had a reduced maximal concentration of ticagrelor $(1156 \pm 771 \mathrm{v}$. $1683 \pm 847 \mathrm{ng} / \mathrm{mL} ; \mathrm{p}=0.006$ ) [14]. Consequently, pharmacodynamic assessment with three different methods showed an attenuated antiplatelet effect of ticagrelor and a greater prevalence of high platelet reactivity in the morphine group [14].
In this sub-analysis, we selected the ratio of total exposure to AR-C124910XX to total exposure to ticagrelor over time as a potential marker which may indicate the difference in conversion of ticagrelor to its active metabolite between the morphine and placebo arms.

The IMPRESSION study was approved by the Ethics Committee of the Nicolaus Copernicus University in Toruń, Collegium Medicum in Bydgoszcz (study approval reference number KB 111/2014) and was conducted in accordance with Good Clinical Practice guidelines, and the principles contained in the Declaration of Helsinki. It was also registered within ClinicalTrials.gov, a registry and results database of publicly and privately supported clinical studies (ClinicalTrials.gov Identifier: NCT02217878). All enrolled patients provided written informed consent to participate in the study.

\section{Endpoints}

The primary endpoint of this sub-analysis was the ratio of $A U C_{(0-12)}$ for $A R-C 124910 X X$ to $A U C_{(0-12)}$ for ticagrelor expressed as a percentage.

\section{Blood sampling}

Blood samples were drawn at eight pre-defined time points according to the blood sampling schedule (pre-loading dose of ticagrelor and $30 \mathrm{~min}, 1 \mathrm{~h}, 2 \mathrm{~h}, 3 \mathrm{~h}$, $4 \mathrm{~h}, 6 \mathrm{~h}, 12 \mathrm{~h}$ post loading dose) [15]. Blood samples for a pharmacokinetic evaluation were collected through a venous cannula (18G) inserted into a forearm vein and the first 3-5 $\mathrm{mL}$ of blood were discarded, mainly to avoid spontaneous platelet activation which could affect the pharmacodynamic outcomes. Immediately after collection, blood samples were centrifuged at 1500 revolutions per minute for 15 minutes. Subsequently, plasma samples were stored at $-80^{\circ} \mathrm{C}$ until the pharmacokinetic analysis.

\section{Pharmacokinetic assessment}

Plasma concentrations of ticagrelor and AR-C124910XX were determined using liquid chromatography coupled with tandem mass spectrometry in the Department of Medicinal Chemistry, Collegium Medicum in Bydgoszcz, Nicolaus Copernicus University in Toruń, Poland. Lower limits of quantification were $4.69 \mathrm{ng} / \mathrm{mL}$ for both ticagrelor and AR-C124910XX [14].

\section{Statistical analysis}

Statistical calculations were performed using the Statistica 12.5 package (StatSoft, Tulsa, OK, USA) and MedCalc 12.0 (MedCalc Software, Mariakerke, 
Belgium) statistical software. Pharmacokinetic calculations were performed using the Matlab R2014 software (Mathworks, Natick, MA, USA). The Shapiro-Wilk test was used to demonstrate whether the investigated continuous variables were normally distributed. Therefore, continuous variables with and without normal distribution were presented as mean values \pm standard deviations or as medians and their interquartile ranges, respectively. The trapezoidal rule was applied to calculate AUC. Depending on the presence or absence of the normal distribution, continuous variables were compared between the study arms with the Student's t-test for independent samples or the Mann-Whitney U-test. Independent categorical variables were compared using the $\chi^{2}$ test with the Yates' correction if required. Linear regression analysis and the Spearman rank correlation coefficients were applied to evaluate the association between $\mathrm{AUC}_{(0-12)}$ for AR-C124910XX and $\mathrm{AUC}_{(0-12)}$ for ticagrelor. Two sided $\mathrm{p}$-values $<0.05$ were considered significant.

\section{Results}

Baseline characteristics of the IMPRESSION study population were well balanced between both study arms (Tab. 1).
The administration of morphine as compared with placebo led to a lower total exposure to both ticagrelor $\left(\mathrm{AUC}_{(0-12)}: 6307 \pm 4359\right.$ v. $9791 \pm 5136 \mathrm{ng} \times \mathrm{h} / \mathrm{mL}$; $\mathrm{p}=0.003)$ and its active metabolite AR-C124910XX $\left(A \cup C_{(0-12)}: 1503 \pm 1138\right.$ v. $2388 \pm 1555 \mathrm{ng} \times \mathrm{h} / \mathrm{mL}$; $\mathrm{p}=0.008$ ) within the first $12 \mathrm{~h}$ after the administration of the $180 \mathrm{mg}$ ticagrelor loading dose [14].

Importantly, the conversion of ticagrelor to its active metabolite within the first $12 \mathrm{~h}$ after the loading dose of ticagrelor, as measured by the ratio of $A \cup C_{(0-12)}$ for AR-C124910XX to $\mathrm{AUC}_{(0-12)}$ for ticagrelor, was comparable in patients assigned to the morphine and placebo arms (20.9 [13.9-34.6] v. 24.7 [18.1-29.6] \%; $p=0.58$ ) (Fig. 1). It is worthy of note that three patients in the morphine arm had $A C_{(0-12)}$ for AR-C124910XX of $0 \mathrm{ng} \times \mathrm{h} / \mathrm{mL}$, and subsequently the ratio of AR-C124910XX exposure to ticagrelor exposure was $0 \%$. In all those cases ticagrelor was not detected in any of the blood samples until the measurement point at $12 \mathrm{~h}$ after the loading dose of ticagrelor, and even then it was present in minimal concentrations only, which seems to be a reasonable explanation for the lack of AR-C124910XX in blood samples obtained within the first $12 \mathrm{~h}$ in those subjects.

Notably, visual inspection of the relationship between $\mathrm{AUC}_{(0-12)}$ for AR-C124910XX and $\mathrm{AUC}_{(0-12)}$ for ticagrelor revealed that regression lines for the morphine and placebo groups were located closely to each other,

Table 1. Baseline characteristics of the study patients

\begin{tabular}{lccc}
\hline \multirow{2}{*}{ Variable } & Morphine & Placebo & p-value \\
\cline { 2 - 3 } Age (years) & $\mathbf{( n = 3 5 )}$ & $\mathbf{( n = 3 5 )}$ & 0.47 \\
Female & $60.7 \pm 10.5$ & $62.5 \pm 10.5$ & 0.19 \\
Body mass index $\left[\mathrm{kg} / \mathrm{m}^{2}\right]$ & $12(34 \%)$ & $7(20 \%)$ & 0.87 \\
STEMI & $27.6 \pm 4.3$ & $27.4 \pm 4.0$ & 0.45 \\
NSTEMI & $24(69 \%)$ & $21(60 \%)$ & 0.45 \\
Metoclopramide use & $11(31 \%)$ & $14(40 \%)$ & $\mathrm{n} / \mathrm{a}$ \\
Hypertension & $1(3 \%)$ & $0(0 \%)$ & 0.15 \\
Diabetes mellitus & $15(43 \%)$ & $21(60 \%)$ & 0.36 \\
Dyslipidemia & $8(23 \%)$ & $5(14 \%)$ & $\mathrm{n} / \mathrm{a}$ \\
Current smoker & $30(86 \%)$ & $31(89 \%)$ & 0.47 \\
Prior AMl & $17(55 \%)$ & $14(45 \%)$ & 0.20 \\
Prior PCl & $5(14 \%)$ & $8(23 \%)$ & 0.12 \\
Prior CABG & $4(11 \%)$ & $9(26 \%)$ & $\mathrm{n} / \mathrm{a}$ \\
Peripheral arterial disease & $0(0 \%)$ & $0(0 \%)$ & 0.31 \\
Chronic renal disease & $3(9 \%)$ & $1(3 \%)$ & 0.31 \\
\hline AMI- & $1(3 \%)$ & $2(6 \%)$ & \\
\hline
\end{tabular}

AMI - acute myocardial infarction; CABG — coronary artery bypass grafting; n/a — not applicable; NSTEMI — non-ST-segment elevation myocardial infarction; $\mathrm{PCl}$ - percutaneous coronary intervention; STEMI - ST-segment elevation myocardial infarction 


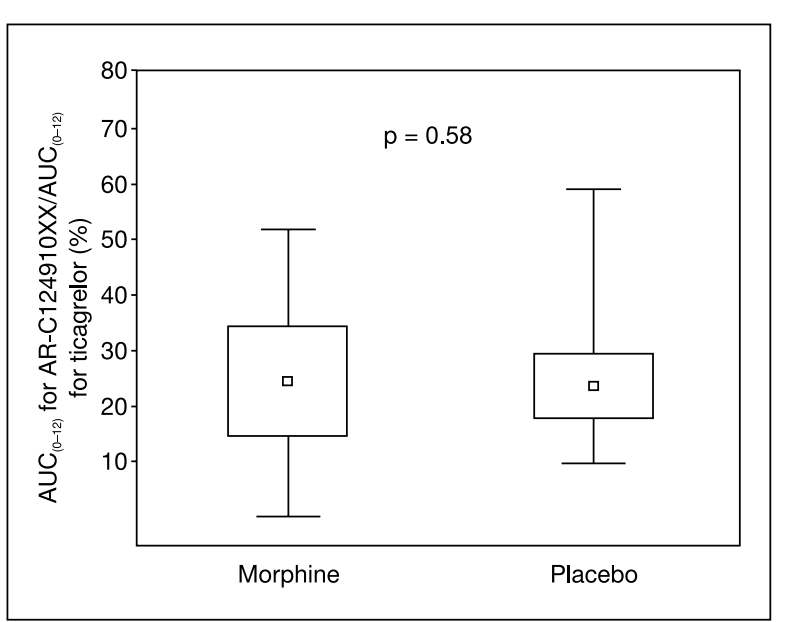

Figure 1. Comparison of the ratios of AR-C124910XX exposure to ticagrelor exposure during the first $12 \mathrm{~h}$ after the administration of a $180 \mathrm{mg}$ ticagrelor loading dose in the morphine and placebo groups. Boxes and whiskers represent medians, interquartile ranges and extreme values

with a tendency for superimposing (Fig. 2). Additionally, we observed similar values of slope coefficients for both study arms in the linear regression equations illustrating the relationship between $\mathrm{AUC}_{(0-12)}$ for AR-C124910XX and $\mathrm{AUC}_{(0-12)}$ for ticagrelor (morphine-treated patients: $\mathrm{AUC}_{(0-12)}$ for AR-C124910XX $=0.19$ $( \pm 0.03) \times \mathrm{AUC}_{(0-12)}$ for ticagrelor +317.99 ( \pm 240.65$)$; $p$ for the slope coefficient $<0.0001$; morphine-naive patients: $A C_{(0-12)}$ for AR-C124910XX $=0.21$ $( \pm 0.04) \times \mathrm{AUC}_{(0-12)}$ for ticagrelor +363.70 ( \pm 424.57$)$; $\mathrm{p}$ for the slope coefficient $<0.0001)$. Strong positive correlations between the $\mathrm{AUC}_{(0-12)}$ for AR-C124910XX and $A \cup C_{(0-12)}$ for ticagrelor were present in both morphine- and placebo-pretreated patients (morphine arm: $R_{S}=0.75 ; p$ for the statistical significance of the $R_{S}$ correlation coefficient < 0.05; placebo arm: $R_{S}=0.79$; $p$ for the statistical significance of the $R_{S}$ correlation coefficient $<0.05$ ), without any statistically significant difference between the values of the Spearman rank correlation coefficients $(p=0.69)$.

\section{Discussion}

The results of this post hoc analysis indicate that within the first $12 \mathrm{~h}$ after the administration of the ticagrelor loading dose, the rate of ticagrelor to AR-C124910XX metabolism is similar in patients presenting with AMI regardless of the morphine administration.

Ticagrelor is predominantly metabolized by cytochrome P450 CYP3A4, however, CYP3A5 enzyme also participates in this process to a lesser extent. The list of compounds known to affect metabolism of ticagrelor

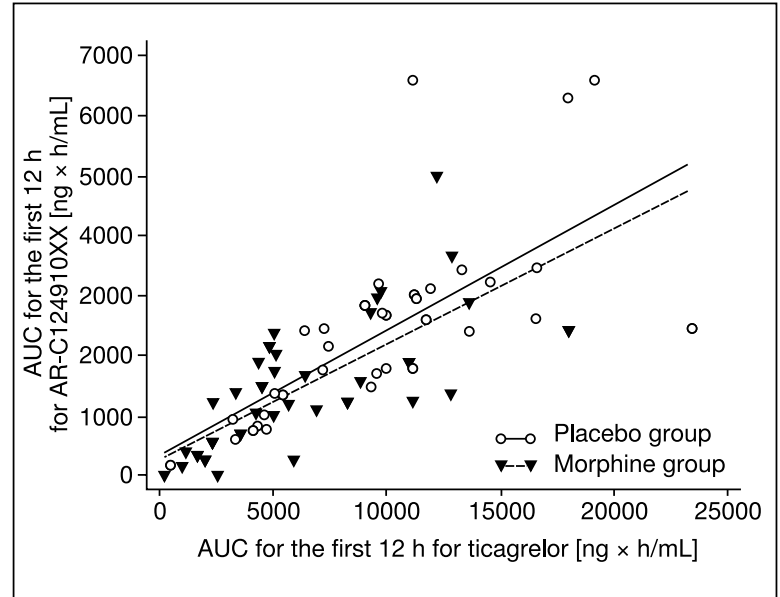

Figure 2. Relationship between $\mathrm{AUC}_{(0-12)}$ for $\mathrm{AR}-\mathrm{C} 124910 \mathrm{XX}$ and $\mathrm{AUC}_{(0-12)}$ for ticagrelor during the first $12 \mathrm{~h}$ after the administration of a $180 \mathrm{mg}$ ticagrelor loading dose in the morphine and placebo groups

includes both CYP3A4 inducers and inhibitors. CYP3A4 inducers (e.g. rifampicin) lead to a lower exposure to this P2Y12 receptor inhibitor by enhancing ticagrelor metabolism [17], which may cause insufficient platelet inhibition and expose patients at increased risk of thrombotic complications [18]. Strong CYP3A4 inhibitors (e.g. ketoconazole) act conversely [19]. Most opioids are metabolized through CYP-mediated oxidation, including CYP3A4, and have significant potential for drug-drug interactions [20]. However, morphine follows a different metabolic pathway and is considered to have relatively smaller burden of CYP-mediated drug interactions [20], which seems to be in line with the results of our sub-analysis.

According to the study by Teng et al., AR-C124910XX, the main active metabolite of ticagrelor, can be found in plasma in the concentration of approximately onethird of the concentration of ticagrelor [8]. In general, the results of our sub-analysis correspond with the previously described pharmacokinetic features of ticagrelor [8]. Importantly, in this study we provide the new insights regarding the ticagrelor-morphine interaction. Briefly, we found that the ratios of $A \cup C_{(0-12)}$ for AR-C124910XX to $A U C_{(0-12)}$ for ticagrelor were comparable in both morphine and placebo pretreated patients. These results are additionally strengthened by the findings coming from the visual inspection of the relationship between $\mathrm{AUC}_{(0-12)}$ for AR-C124910XX and $A \cup C_{(0-12)}$ for ticagrelor, which revealed closely located regression lines for the morphine and placebo groups, with a tendency for superimposing. Moreover, we observed similar values of slope coefficients for both study arms in the linear regression equations illustrating the relationship between $A U C_{(0-12)}$ for AR-C124910XX and $\mathrm{AUC}_{(0-12)}$ for ticagrelor. 
In the light of the current sub-analysis, an attenuation and delay in the exposure to AR-C124910XX observed in the morphine arm of the IMPRESSION study [14] are most likely caused by decreased concentrations of the parent compound, probably resulting from morphine-induced impaired gastric emptying, lower intestinal motility and higher incidence of vomiting. The activation of the opioid receptors located in the myenteric plexus and in the intestines was previously demonstrated to decrease propulsive motility and secretion of the gastro-intestinal tract [13]. The results obtained in this study expand the knowledge regarding the pharmacokinetics of ticagrelor, but also underline the need of elucidation and a detailed examination of the mechanisms underlying the attenuated and delayed exposure to ticagrelor and its active metabolite in AMI patients.

\section{Study limitations}

Several limitations of our study need to be acknowledged. Firstly, the IMPRESSION study was not designed to investigate the detailed mechanisms responsible for decreased plasma concentrations of ticagrelor and its active metabolite in morphine-treated patients. Therefore, the function of cytochrome P450 CYP3A4 and CYP3A5 enzymes, which are involved in ticagrelor's transformation into AR-C124910XX, were not directly examined and our observations are only hypothesis generating. Additionally, this sub-analysis may be underpowered to detect a minor impact of the morphine administration on the conversion of ticagrelor to its active metabolite. Moreover, morphine dosing regimens, different from those employed in the IMPRESSION study, might have a greater influence on ticagrelor's metabolism. Finally, the present sub-analysis was performed post hoc and was not predefined.

\section{Conclusions}

In this sub-analysis of the IMPRESSION study, we did not find any evidence that the extent of ticagrelor conversion to AR-C124910XX is affected by the morphine administration. A decreased exposure to AR-C124910XX in the morphine arm compared with the placebo arm of the IMPRESSION study was most likely caused by a proportional attenuation of the exposure to the parent drug.

\section{Acknowledgements}

Rafał Bilski, Aleksandra Karczmarska-Wódzka, Julia Kubica, Ewa Laskowska, Ewa Obońska, Karolina
Obońska and Joanna Sikora are acknowledged for their collaboration in the conduct of the study.

\section{Study funding}

The IMPRESSION study was entirely funded by Collegium Medicum of the Nicolaus Copernicus University (NCU CM grant no. 202).

\section{Statement of competing interests}

Dr. Jacek Kubica received a consulting fee from AstraZeneca. Dr. Marek Koziński received honoraria for the lectures from AstraZeneca. All other authors have reported that they have no relevant competing interests to disclose.

\section{References}

1. Koziński M, Grześk G, Kubica J. Optimal antiplatelet and antithrombotic therapy in patients with ST elevation myocardial infarction. Kardiol Pol 2012; 70: 206-212.

2. Navarese EP, Verdoia M, Schaffer $A$ et al. Ischaemic and bleeding complications with new, compared to standard, ADP-antagonist regimens in acute coronary syndromes: a meta-analysis of randomized trials. QJM 2011; 104: 561-569.

3. Steg PG, James SK, Atar D et al. ESC Guidelines for the management of acute myocardial infarction in patients presenting with ST-segment elevation. Eur Heart J 2012; 33: 2569-2619.

4. Roffi M, Patrono C, Collet JP et al. 2015 ESC Guidelines for the management of acute coronary syndromes in patients presenting without persistent ST-segment elevation: Task Force for the Management of Acute Coronary Syndromes in Patients Presenting without Persistent ST-Segment Elevation of the European Society of Cardiology (ESC). Eur Heart J 2015. doi:10.1093/eurheartj/ehv320 [Epub ahead of print].

5. Wallentin L, Becker RC, Budaj A et al. Ticagrelor versus clopidogrel in patients with acute coronary syndromes. N Engl J Med 2009; 361: 1045-1057.

6. Navarese EP, Buffon A, Kozinski M et al. A critical overview on ticagrelor in acute coronary syndromes. QJM 2013; 106: 105-115.

7. Husted S, Emanuelsson H, Heptinstall S, Sandset PM, Wickens M Peters G. Pharmacodynamics, pharmacokinetics, and safety of the oral reversible P2Y12 antagonist AZD6140 with aspirin in patients with atherosclerosis: a double-blind comparison to clopidogrel with aspirin. Eur Heart J 2006; 27: 1038-1047.

8. Teng R, Oliver S, Hayes MA, Butler K. Absorption, distribution, metabolism, and excretion of ticagrelor in healthy subjects. Drug Metab Dispos 2010; 38: 1514-1521.

9. Teng R, Butler K. Pharmacokinetics, pharmacodynamics, tolerability and safety of single ascending doses of ticagrelor, a reversibly binding oral P2Y(12) receptor antagonist, in healthy subjects. Eur J Clin Pharmacol 2010; 66: 487-496.

10. Husted SE, Storey RF, Bliden $\mathrm{K}$ et al. Pharmacokinetics and pharmacodynamics of ticagrelor in patients with stable coronary artery disease: results from the ONSET-OFFSET and RESPOND studies. Clin Pharmacokinet 2012; 51: 397-409.

11. Storey RF, Husted S, Harrington RA et al. Inhibition of platelet aggregation by AZD6140, a reversible oral P2Y12 receptor antagonist, compared with clopidogrel in patients with acute coronary syndromes. J Am Coll Cardiol 2007; 50: 1852-1856.

12. Parodi G. Chest pain relief in patients with acute myocardial infarction. Eur Heart J Acute Cardiovasc Care 2015. doi:10.1177/2048872615584078 [Epub ahead of print].

13. Holzer P. Opioid receptors in the gastrointestinal tract. Regul Pept 2009; 155: 11-17 
14. Kubica J, Adamski P, Ostrowska $\mathrm{M}$ et al. Morphine delays and attenuates ticagrelor exposure and action in patients with myocardial infarction: the randomized, double-blind, placebo-controlled IMPRESSION trial. Eur Heart J 2015. doi:10.1093/eurheartj/ehv547 [Epub ahead of print]

15. Kubica J, Adamski P, Ostrowska M et al. Influence of Morphine on Pharmacokinetics and Pharmacodynamics of Ticagrelor in Patients with Acute Myocardial Infarction (IMPRESSION): study protocol for a randomized controlled trial. Trials 2015; 16: 198

16. Thygesen K, Alpert JS, Jaffe AS et al. Third universal definition of myocardial infarction. Eur Heart J 2012; 33: 2551-2567.
17. Teng R, Mitchell P, Butler K. Effect of rifampicin on the pharmacokinetics and pharmacodynamics of ticagrelor in healthy subjects. Eur J Clin Pharmacol 2013; 69: 877-883

18. Aradi D, Kirtane A, Bonello L et al. Bleeding and stent thrombosis on P2Y12-inhibitors: collaborative analysis on the role of platelet reactivity for risk stratification after percutaneous coronary intervention. Eur Heart J 2015; 36: 1762-1771.

19. Teng R, Butler K. Effect of the CYP3A inhibitors, diltiazem and ketoconazole, on ticagrelor pharmacokinetics in healthy volunteers. J Drug Assess 2013; 2: 30-39.

20. Smith HS. Opioid Metabolism. Mayo Clin Proc 2009; 84: 613-624. 